\title{
Comparison of Ambulatory Tonometric and Oscillometric Blood Pressure Monitoring in Hypertensive Patients
}

This article was published in the following Dove Press journal: Integrated Blood Pressure Control

\section{Bodil Gade Hornstrup (iD Jeppe Bakkestrøm Rosenbæk (D) Jesper Nørgaard Bech (D)}

University Clinic in Nephrology and Hypertension, Regional Hospital West Jutland and Aarhus University, Holstebro, Denmark
Correspondence: Bodil Gade Hornstrup University Clinic in Nephrology and Hypertension, Regional Hospital West Jutland and Aarhus University, Holstebro, Denmark

Email bodil.hornstrup@rm.dk
Aim: Correct measurement of blood pressure (BP) is important for optimal diagnosis and treatment of patients with hypertension. The aim of this study was to compare a wrist-worn device using tonometric measurements of BP to a conventional device using oscillometric measurements of $24 \mathrm{~h} \mathrm{BP}$, diagnosing of hypertension, and non-dipping.

Methods: One-hundred patients in the Renal Outpatient Clinic had $24 \mathrm{~h}$ ambulatory BP monitoring performed with a tonometric device, BPro, and an oscillometric device, A\&D, simultaneously. Results: Twenty-four-hour and daytime systolic BP was significantly lower using tonometric monitoring compared to oscillometric ( 7 and $6 \mathrm{mmHg}$, respectively, $p<0.001$ ). In the population of patients diagnosed with hypertension, the tonometric device diagnosed $90 \%$ of patients with uncontrolled hypertension correctly (positive predictive value), whereas $49 \%$ of patients classified as normotensive were uncontrolled hypertensive (negative predictive value). The mean difference between relative nocturnal BP decrease between tonometric and oscillometric was $2 \pm 8 \%$ ( $p<0.01$ ), and $33 \%$ of patients classified as dippers were nondippers (negative predictive value).

Conclusion: Using the BPro device for tonometric monitoring of BP and classification of hypertension and non-dipping in patients diagnosed with hypertension leads to misclassification of patients. Therefore, the BPro device is not suitable for clinical practice in hypertensive patients from a Renal Outpatient Clinic.

Keywords: ambulatory blood pressure monitoring, oscillometric, tonometric

\section{Plain Language Summary}

High blood pressure is the main contributor to morbidity and mortality related to cardiovascular disease, i.e. stroke, heart failure, and renal disease. Optimal treatment of patients with high blood pressure relies on correct monitoring of blood pressure.

Blood pressure is commonly measured with an oscillometric device. That is a device with an inflatable cuff around the upper arm. One study has proposed that this technique is less accurate in patients with stiff arteries, which can be seen in patients with chronic renal failure or diabetes.

The tonometric technique is another way to measure blood pressure. This technique is based on capturing pulse wave reflections in the arterial surface, and blood pressure is calculated from this using an algorithm. One device using this technique is the BPro ${ }^{\circledR}$ device shaped like a wrist-worn watch. BPro has never been compared to the commonly used oscillometric devices in patients from a Renal Outpatient Clinic. Therefore, the aim of this study was to investigate if BPro diagnosed high blood pressure correctly in patients from a Renal Outpatient Clinic. 
We compared blood pressure levels in 100 patients, and we found that blood pressure levels measured with BPro were significantly lower than oscillometric blood pressure levels, and patients were misclassified as having normal blood pressure when blood pressure was, in fact, high and vice versa. Therefore, we did not find the BPro device suitable for monitoring of blood pressure control in this population, because it would lead to wrong treatment of patients with high blood pressure.

\section{Introduction}

Hypertension is accepted as the main contributor to cardiovascular $(\mathrm{CV})$ related morbidity and mortality. ${ }^{1-3}$ High blood pressure (BP) is related to around $50 \%$ of the cases of strokes and ischemic heart disease, ${ }^{1}$ and the risk of cardiovascular disease (CVD) rises from office BP levels as low as $115 / 75 \mathrm{mmHg} .{ }^{4}$ Optimal treatment of hypertensive patients relies on correct monitoring of BP. Studies have shown that $24 \mathrm{~h}$ ambulatory BP monitoring (ABPM) is more accurate than office $\mathrm{BP}$ to predict the risk of $\mathrm{CV}$ morbidity and mortality, 5,6 and today nocturnal BP and non-dipping status are considered to possess a stronger correlation to the risk of CVD than daytime $\mathrm{BP}^{7-9}$

Oscillometric devices are widely used in daily clinical practice. One study has shown oscillometric devices to be less accurate in patients with increased arterial stiffness. ${ }^{10}$ In contrast to the oscillometric device, the tonometric device uses another technique, where pulse wave reflections are captured from the arterial surface, and blood pressure is calculated using an algorithm. The BPro ${ }^{\circledR}$ device used in the present study has previously been validated, and it met the accuracy criteria of the Association for the Advancement of Medical Instrumentation standards. ${ }^{11}$ However, this validation did not include $24 \mathrm{~h}$ measurements and did not include patients with renal disease. The aim of the present study was to analyze whether peripheral BP measured by BPro $^{\circledR}$ using the tonometric technique was comparable to brachial BP measured by A\&D device using an oscillometric technique in a population of patients in the Renal Outpatient Clinic, Regional Hospital West Jutland, Denmark.

\section{Materials and Methods}

\section{Study Population}

From December 2012 to June 2015 patients were recruited from the Renal Outpatient Clinic, Regional Hospital West Jutland. Inclusion criteria were men and women aged 18-90 years. Exclusion criteria were $>10 \mathrm{mmHg}$ difference in BP between right and left arm, atrial fibrillation, and unwillingness to participate.

\section{Ethics}

A request for approval from The Regional Committees on Health Research Ethics, Denmark, was sent. The Committee did not find it to be a trial as defined in the Announcement of the Act on Scientific Ethical Treatment of Health Science Research Projects, and the committee did, therefore, not need to approve this study.

\section{Procedure}

If the patient met the inclusion criteria at the time for a pre-booked conventional $24 \mathrm{~h} \mathrm{ABPM}$ in the Renal Outpatient Clinic, they were invited to participate. If the patient accepted, the tonometric device was placed simultaneously with the oscillometric device, following the procedure as written below. Patients did not need to provide written informed consent. Data of medical prescriptions and levels of $\mathrm{p}$-creatinine and u-albumin were derived from the electronic patient record system.

\section{Tonometric Blood Pressure}

Tonometric BP was measured by applanation tonometry using BPro $^{\circledR}$ (HealthSTATS, International Pte. Ltd., Singapore) placed on the radial artery on the left wrist. The BPro device was calibrated with a mean of the last three of four BP measured on the left arm with the A\&D device, later used for $24 \mathrm{~h}$ measurement on the same subject. BP was measured every $15 \mathrm{~min}$ during $24 \mathrm{~h}$. Daytime was fixed at 6.01 am to $10.59 \mathrm{pm}$, nighttime was fixed at $11 \mathrm{pm}$ to $6 \mathrm{am}$. We did not include a limit of measurements for accepting the tonometric ABPM.

\section{Oscillometric Blood Pressure}

Oscillometric BP was measured using A\&D TM-2430 (A\&D Company Limited, Tokyo, Japan) with a cuff placed on the right upper arm. The upper arm's circumference was measured, and an appropriate size cuff was applied. BP was measured every 20 min throughout $24 \mathrm{~h}$. Day- and nighttime was fixed as for the tonometric device, and as for the tonometric device, we did not include a limit of measurements for accepting the ABPM.

\section{Definition of Hypertension}

Hypertension was defined as brachial $24 \mathrm{~h}$ BP $\geq 130$ $\mathrm{mmHg}$ systolic and/or $\geq 80 \mathrm{mmHg}$ diastolic. Non-dipping was defined as a relative nocturnal systolic BP decrease $\leq 10 \%$. These definitions are according to the most recent 
guidelines from the European Society of Hypertension/ European Society of Cardiology. ${ }^{12}$

Uncontrolled hypertension was defined as brachial $24 \mathrm{~h} \mathrm{BP} \geq 130 \mathrm{mmHg}$ systolic and/or $\geq 80 \mathrm{mmHg}$ diastolic despite the awareness of hypertension diagnosis. Isolated systolic hypertension was defined as brachial 24 $\mathrm{h} \mathrm{BP} \geq 130 \mathrm{mmHg}$ systolic and $<80 \mathrm{mmHg}$ diastolic.

\section{Biochemical Analyses}

P-creatinine and urinary concentrations of albumin were measured by routine methods at the Department of Clinical Biochemistry, Regional Hospital West Jutland, Denmark. eGFR was calculated using the CKD-MDRD equation.

\section{Statistical Methods}

Statistical analyses were performed by the authors using IBM SPSS statistics version 22.0 (IBM Corp.; Armonk, NY, United States). All data were tested for normality and variance equality. The statistical level of significance was $p<0.05$ in all analyses. Unless otherwise stated, normally distributed continuous variables were reported as means with standard deviation. Paired $t$-test was used for parametric continuous variables and Wilcoxon test for non-parametric continuous data. Correlation analyses on normally distributed continuous variables were performed with Pearson's test and with interclass correlation coefficient (ICC). Categorical variables were reported as percentages with number. McNemar's test was used to determine if any difference on a dichotomous dependent variable between two groups exited, while Cohen's kappa (к) concordance test was used to test for agreement between the two paired categorical variables.

Positive and negative predictive values were calculated using these formulas: positive predictive value (PPV) = number of true positives/total number of positives and negative predictive value $(\mathrm{NPV})=$ number of true negatives/total number of negatives.

\section{Results}

\section{Study Participants}

The study included 100 patients, 38 women and 62 men. The mean age was $59 \pm 14$ years, and mean BMI was $28 \pm 6 \mathrm{~kg} / \mathrm{m}^{2}$. All patients were Caucasians. Eighty-three patients received antihypertensive treatment, and all patients but two had hypertension and/or received antihypertensive treatment. Twenty patients received anti-diabetic medication. Twentynine patients received aspirin as antithrombotic treatment. Forty-one patients received statins. Mean eGFR was 60 $\pm 31 \mathrm{~mL} / \mathrm{min} / 1.73 \mathrm{~m}^{2}$, total range [10-155] $\mathrm{mL} / \mathrm{min} / 1.73 \mathrm{~m}^{2}$. Forty patients had albuminuria (u-albumin $>30 \mathrm{mg} / \mathrm{L}$ ).

\section{Quantity of ABPM}

Oscillometric ABPM consisted of a mean of 83 (total range 55-106) measurements with 65 daytime (total range 41-83) and 18 nighttime (total range 5-28). Tonometric ABPM consisted of a mean of 56 (total range 23-81) measurements with 36 (total range 17-61) daytime and 20 (total range 6-28) nighttime measurements.

\section{Blood Pressure}

The difference in BP between right and left arm was nonsignificant (SBP -0.95 and DBP $-0.03, p>0.05$ ).

Oscillometric and tonometric $24 \mathrm{~h}$, daytime, and nighttime BP values are displayed in Table 1 along with mean difference. Oscillometric systolic $24 \mathrm{~h}$, daytime measurements and relative nocturnal BP decrease were significantly higher than tonometric measurements, whereas nighttime oscillometric DBP was significantly lower. There was no significant difference between oscillometric and tonometric $24 \mathrm{~h}$ and daytime DBP and nighttime SBP.

Table I Oscillometric and Tonometric Blood Pressure Measurements

\begin{tabular}{|l|l|l|l|l|}
\hline & Oscillometric & Tonometric & Mean Difference & $\boldsymbol{P}$ \\
\hline $24 \mathrm{~h} \mathrm{SBP}, \mathrm{mmHg}$ & $136(15)$ & $129(19)$ & $7(17)$ & $<0.000 \mathrm{I}$ \\
$24 \mathrm{~h} \mathrm{DBP}, \mathrm{mmHg}$ & $78(8)$ & $80(13)$ & $-1(10)$ & 0.19 \\
Daytime SBP, $\mathrm{mmHg}$ & $139(14)$ & $133(20)$ & $6(17)$ & $<0.001$ \\
Daytime DBP, mmHg & $81(8)$ & $82(13)$ & $-2(10)$ & 0.07 \\
Nighttime SBP, mmHg & $124(19)$ & $122(18)$ & $3(20)$ & 0.16 \\
Nighttime DBP, mmHg & $70(10)$ & $75(13)$ & $-6(10)$ & $<0.0001$ \\
Relative nocturnal SBP decrease, \% & $10(8)$ & $8(6)$ & $2(8)$ & 0.01 \\
\hline
\end{tabular}

Notes: Data are presented as mean (SD). Mean difference $=$ oscillometric - tonometric values.

Abbreviations: SBP, systolic blood pressure; DBP, diastolic blood pressure; SD, standard deviation. 


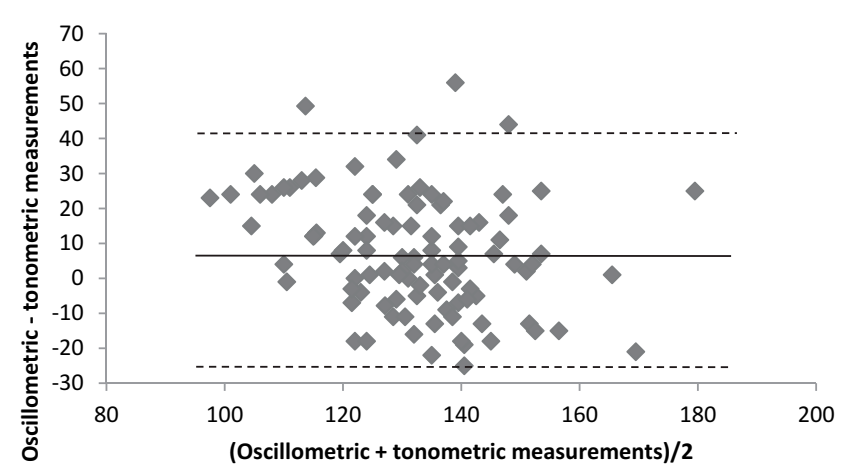

Figure I Bland-Altman plot presenting the difference in blood pressure $(\mathrm{mmHg})$ between tonometric and oscillometric $24 \mathrm{~h}$ systolic measurements. The solid black line represents the mean difference and the dotted lines represent mean difference $\pm 2 S D$.

Bland-Altman plot was used to graphically present the difference between oscillometric and tonometric measurements plotted against the averages of the two measurements. Figure 1 shows a Bland-Altman plot for $24 \mathrm{~h}$ SBP measurements. The mean difference between tonometric and oscillometric measurements was above zero, depicted as the solid black line in Figure 1. This shows a systematic difference between tonometric and oscillometric measurements over the entire range of SBP values. The tonometric measurements were equally higher and lower than the oscillometric measurements at every measured BP level, and ninety-five percent of the measurements is within \pm 2SD limit of the mean difference.

Figure 2 shows Bland-Altman plot for relative nocturnal SBP decrease. Also in this plot, there is a systematic difference between the relative decreases between tonometric and oscillometric measurements over the entire range of relative decrease values with equally higher and lower tonometric than oscillometric values. Between $90 \%$

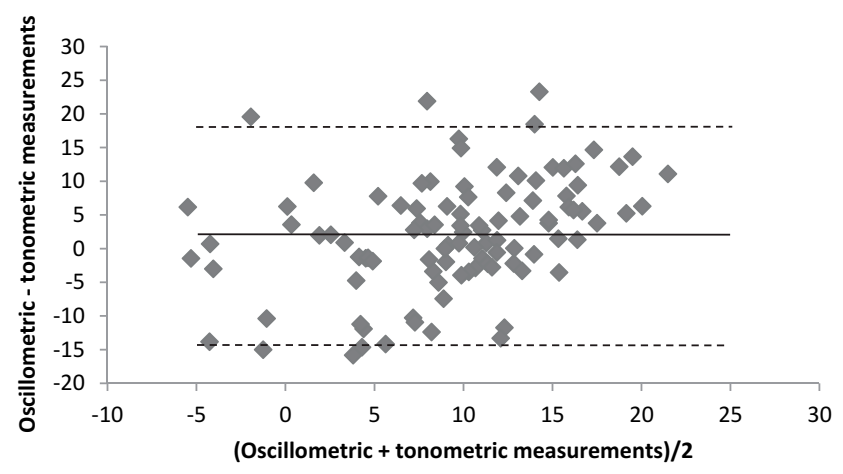

Figure 2 Bland-Altman plot presenting the difference in relative nocturnal systolic blood pressure (\%) between tonometric and oscillometric measurements. The solid black line represents the mean difference and the dotted lines represent mean difference $\pm 2 S D$. and $95 \%$ of measurements are within the $\pm 2 \mathrm{SD}$ range from the mean difference.

Correlation coefficients from linear correlation analyses between oscillometric and tonometric BP measurements are seen in Table 2 along with ICC. There were significant correlations between all parameters with p-values $<0.0001$ using the Pearson's test. ICC was $>0.90$ with regard to $24 \mathrm{~h} \mathrm{SBP}$ and between 0.50 and 0.75 for the other ABPM parameters, while ICC was $<0.5$ for relative nocturnal BP decrease. All parameters were significant with a wide $95 \% \mathrm{CI}$.

\section{Prediction of Hypertension, Uncontrolled Hypertension, and Non-Dipping}

Ninety-eight patients were previously diagnosed with hypertension as mentioned above. Including these 98 patients in analyses, uncontrolled hypertension as defined above was seen in 73 patients using oscillometric $24 \mathrm{~h}$ measurements and 60 patients using tonometric $24 \mathrm{~h}$ measurements (McNemar's test $p=0.019, \mathrm{~K}=0.405(p<0.00001)$ ).

In the 98 patients diagnosed with hypertension, the positive predictive value (PPV) of uncontrolled hypertension using the tonometric BPro with oscillometric $24 \mathrm{~h}$ measurements as diagnostic standard was $90 \%$, whereas the negative predictive value (NPV) of uncontrolled hypertension using the tonometric device was 49\%. The corresponding PPV and NPV for diagnosing hypertension in the whole population were $88 \%$ and $50 \%$, respectively.

Forty-four patients were non-dippers using oscillometric measurements, and 57 patients were non-dippers using tonometric measurements (McNemar's test $p=0.060$, $\mathrm{K}=0.195(p=0.045))$. The PPV of using the tonometric

Table 2 Correlation Between Oscillometric and Tonometric Blood Pressure Measurements

\begin{tabular}{|l|l|l|}
\hline & $\begin{array}{l}\text { r (Pearson's } \\
\text { Test) }\end{array}$ & ICC (95\% Cl) \\
\hline 24 h SBP & $0.525^{* *}$ & $0.94(0.34 ; 0.98)^{* *}$ \\
24 h DBP & $0.606^{* *}$ & $0.71(0.56 ; 0.80)^{* *}$ \\
Daytime SBP & $0.520^{* *}$ & $0.64(0.45 ; 0.76)^{* *}$ \\
Daytime DBP & $0.608^{* *}$ & $0.70(0.55 ; 0.80)^{* *}$ \\
Nighttime SBP & $0.446^{* *}$ & $0.61(0.43 ; 0.74)^{* *}$ \\
Nighttime DBP & $0.590^{* *}$ & $0.68(0.43 ; 0.8 I)^{* *}$ \\
Relative nocturnal SBP & $0.355^{* *}$ & $0.49(0.24 ; 0.65)^{* *}$ \\
decrease, \% & & \\
\hline
\end{tabular}

Note: $* * p<0.0001$.

Abbreviations: DBP, diastolic blood pressure; ICC, intraclass correlation; SBP, systolic blood pressure. 
$24 \mathrm{~h}$ measurements to classify non-dippers with oscillometric measurements as diagnostic standard was 0.53 or $53 \%$, which means $53 \%$ of patients classified as nondippers using tonometric measurements were true nondippers, $47 \%$ were dippers. The NPV was 0.67 or $67 \%$, meaning that $67 \%$ of patients classified as dippers using tonometric measurements were true dippers according to oscillometric measurements.

\section{Subgroup Analyses \\ $\mathrm{BMI}$}

Subgroup analyses of the 24 patients with BMI $\geq 30$ showed difference between oscillometric and tonometric $24 \mathrm{~h}$ SBP of 4 (13) $\mathrm{mmHg}(p=0.2)$, daytime SBP of 1 (13) $\mathrm{mmHg}(p=0.6)$, and nighttime SBP 2 (18) $\mathrm{mmHg}(p=0.6)$. Analyses of patients with BMI $<30$ were similar to whole group analyses (data not shown).

\section{Isolated Systolic Hypertension}

Twenty-five patients had isolated systolic hypertension as defined above. In this population, analyses showed the following difference between oscillometric and tonometric measurements: $24 \mathrm{~h}$ SBP of 10 (19) $\mathrm{mmHg}(p=0.01)$, $24 \mathrm{~h} \mathrm{DBP}$ of -2 (10) $\mathrm{mmHg}(p=0.01)$, daytime SBP of 9 (20) $\mathrm{mmHg}(p=0.3)$, daytime DBP of -3 (10) $\mathrm{mmHg}$ $(p=0.2)$, nighttime SBP $9(10) \mathrm{mmHg}(p=0.06)$, and nighttime DBP $-5(10) \mathrm{mmHg}(p=0.016)$.

\section{Patients Treated with Statins}

Sub analyses of $24 \mathrm{~h}$ ABPM parameters in the population of 41 patients receiving statins were similar to whole group analyses (data not shown).

\section{Number of Measurements}

Additional paired $t$-test analyses including only patients with a total number of 55 tonometric measurements or more $(n=63)$ showed similar values to the whole group analyses with regard to mean difference between oscillometric and tonometric mean values on $24 \mathrm{~h}$, daytime, nighttime, and relative nocturnal SBP decrease (data not shown).

\section{Discussion}

The present study found that ambulatory BP measured by a tonometric device, BPro, was lower than oscillometric values with regard to $24 \mathrm{~h}$ and daytime SBP, whereas $24 \mathrm{~h}$ and daytime diastolic values were at a similar level. There was a significant, but weak, correlation between tonometric and oscillometric $24 \mathrm{~h}$, day- and nighttime measurements (significant Pearson correlation coefficient $>0.45$ ). The interclass correlation was excellent with regard to $24 \mathrm{~h} \mathrm{SBP}$, but only fair regard to the other ABPM parameters, whereas ICC of relative nocturnal BP decrease was poor. ${ }^{13}$ There was a systematic difference between tonometric and oscillometric measurements, but more than $95 \%$ of measurements were within 2SD from the mean difference between the two devices when analyzing $24 \mathrm{~h}$ systolic BP. This is within the limits recommended by Bland and Altman. ${ }^{14}$ The PPV and NPV of diagnosing uncontrolled hypertension with the tonometric device were $90 \%$ and $49 \%$, respectively, and which means, that $90 \%$ of patients with uncontrolled hypertension using tonometric monitoring were truly uncontrolled hypertensive, whereas $49 \%$ of patients classified as normotensive were actually uncontrolled hypertensive.

Two previous studies, one study on a small diabetic population $^{15}$ and one study on 50 normo- and prehypertensive subjects ${ }^{16}$ have examined the tonometric BPro device against an oscillometric device and concluded agreement between tonometric and oscillometric measurements. However, the study on diabetic patients only compared office tonometric and oscillometric measurements. The study on normo- and prehypertensive patients did find a significant difference between measurements, as did we.

As proposed by van Popele et al $^{10}$ in 2000 , the overestimation of both systolic and diastolic BP using oscillometric devices may be related to arterial stiffness. If the tonometric device was more accurate in estimating BP, we would have expected lower tonometric SBP and DBP in the diabetic population from the previous study, ${ }^{15}$ and similar tonometric and oscillometric measurements in the normo- and prehypertensive population. ${ }^{16}$ In our study, increased arterial stiffness could explain why systolic BP was higher using oscillometric measurements. However, we would have expected that the difference between oscillometric and tonometric BP levels was higher in patients with isolated systolic hypertension and lower in patients treated with statins than the whole population, but we found the same levels of difference. We did not know the duration and adherence of statin treatment, which may influence the negative finding. However, it is not clear how the degree of arterial stiffness influences the findings of the present study, and how the tonometric device can accommodate the challenge of arterial stiffness.

In the present study, we excluded patients with more than $10 \mathrm{mmHg}$ difference between arms, and there was a non-significant inter-arm difference of less than 1 
mmHg. Therefore, we do not consider this as a possible explanation for the difference between methods.

Sub analyses of the patients with $\mathrm{BMI} \geq 30$ showed a non-significant difference between tonometric and oscillometric measurements. This suggests that the tonometric device may be useful on this patient population, or maybe the non-significant difference is related to the smaller patient population and therefore lack of statistical power.

It is relevant to analyze if the quantity of measurements can be a source of difference between results from two devices. In the present study, we found that oscillometric ABPM had more daytime measurements but a similar number of nighttime measurement compared to tonometric measurements. However, we did subanalyses on the patients with more than 55 tonometric measurements, which was the lowest number of total oscillometric measurements over $24 \mathrm{~h}$, and these subanalyses were similar to whole group analyses.

In our study, we did not have a minimally acceptable number of measurements for accepting the ABPM. According to a study from Agarwal et al from 2018, ${ }^{17}$ means from 8 randomly selected systolic BP measurements from a full $24 \mathrm{~h}$ ABPM were in good agreement with the mean from the full $24 \mathrm{~h}$ measurements. According to this finding, all 100 patients had an acceptable number of measurements from both devices.

The relative nocturnal SBP decrease was significantly lower using tonometric measurements compared with oscillometric measurements, and the correlation between the two measurements of the relative decrease was poor, but significant. The Bland-Altman plot revealed a small systematic difference between tonometric and oscillometric measurements, and less than the recommended $95 \%$ of measurements were within the 2SD limit. Therefore, using the tonometric BPro device to assess the degree of dipping is related to inaccuracy. The PPV of diagnosing non-dipping using tonometric measurements was $53 \%$, which means that $47 \%$ of patients diagnosed as non-dippers with the tonometric device were actually dippers. The NPV was $67 \%$, which means that when using the tonometric device $33 \%$ of patients classified as dippers were non-dippers. In the present study, the tonometric BPro device underestimated the degree of dipping and led to misclassification of dipping status.

Current guidelines of diagnosing and treatment of hypertension are based on BP measured by oscillometric devices. Using tonometric techniques, therefore, needs studies analyzing the effect on patients' health complications and survival, when using tonometric devices instead of wellknow oscillometric devices.

\section{Strengths and Limitations}

It is a strength in this study that we used the oscillometric device for $24 \mathrm{~h}$ measurements for calibration of the tonometric device and thereby eliminated an extra source of difference between measurements. Moreover, there were a high number of measurements using both the oscillometric and tonometric device. The BP difference between right and left arm was non-significant, and only patients with less than $10 \mathrm{mmHg}$ BP difference were included.

It is a weakness that we did not repeat the $24 \mathrm{~h}$ monitoring to test the reproducibility of the difference between oscillometric and tonometric measurements. Using this type of tonometric BP measuring device, calibration relies on the oscillometric measurement of BP, which may challenge the findings.

\section{Conclusion}

From the present study, we do not find the BPro tonometric device suitable for evaluating BP in hypertensive patients from a Renal Outpatient Clinic. Despite the significant correlation between oscillometric and tonometric measurements, the use of tonometric measurements leads to misclassification of hypertension and dipping status.

\section{Abbreviations}

ABPM, ambulatory blood pressure monitoring; BMI, Body mass index; BP, blood pressure; CV, cardiovascular; CVD, cardiovascular disease; DBP, diastolic blood pressure; eGFR, estimated glomerular filtration rate; h, hour; ICC, Intra-Class Correlation; NPV, negative predictive value; PPV, positive predictive value; SBP, systolic blood pressure.

\section{Acknowledgments}

The authors acknowledge the staff in the Renal Outpatients Clinic, Regional Hospital West Jutland, for the help in conducting this study.

\section{Funding}

This research project did not receive any research funding.

\section{Disclosure}

All authors declare that they have no competing interests. The authors alone are responsible for the content and writing of the paper. 


\section{References}

1. Lawes CMM, Vander Hoorn S, Rodgers A. Global burden of blood-pressure-related disease, 2001. Lancet (London, England). 2008;371(9623):1513-1518. doi:10.1016/S0140-6736(08)60655-8

2. Ezzati M, Lopez AD, Rodgers A, Vander Hoorn S, Murray CJ. Selected major risk factors and global and regional burden of disease. Lancet. 2002;360(9343):1347-1360. doi:10.1016/S0140-67 36(02)11403-6

3. Whitworth JA; World Health Organization, International Society of Hypertension Writing Group. 2003 World Health Organization (WHO)/International Society of Hypertension (ISH) statement on management of hypertension. J Hypertens. 2003;21(11):1983-1992.

4. Lewington S, Clarke R, Qizilbash N, Peto R, Collins R; Prospective Studies Collaboration. Age-specific relevance of usual blood pressure to vascular mortality: a meta-analysis of individual data for one million adults in 61 prospective studies. Lancet. 2002;360 (9349):1903-1913. doi:10.1016/S0140-6736(02)11911-8

5. Perloff D, Sokolow M, Cowan R. The prognostic value of ambulatory blood pressures. JAMA. 1983;249(20):2792-2798. doi:10.1001/ jama.1983.03330440030027

6. Drayer JIM, Weber MA, DeYoung JL. BP as a determinant of cardiac left ventricular muscle mass. Arch Intern Med. 1983;143(1):90. doi:10.1001/archinte.1983.00350010094016

7. Staessen JA, Thijs L, Fagard R, et al. Predicting cardiovascular risk using conventional vs ambulatory blood pressure in older patients with systolic hypertension. Systolic Hypertension in Europe Trial Investigators. JAMA. 1999;282(6):539-546. doi:10.1001/jama.282.6.539

8. Dolan E, Stanton A, Thijs L, et al. Superiority of ambulatory over clinic blood pressure measurement in predicting mortality: the Dublin outcome study. Hypertension. 2005;46(1):156-161. doi:10.1161/01. HYP.0000170138.56903.7a
9. Muxfeldt ES, Cardoso CR, Salles GF. Prognostic value of nocturnal blood pressure reduction in resistant hypertension. Arch Intern Med. 2009;169(9):874-880. doi:10.1001/archinternmed.2009.68

10. van Popele NM, Jan Bos WW, de Beer NA, et al. Arterial stiffness as underlying mechanism of disagreement between an oscillometric blood pressure monitor and a sphygmomanometer; 2000. Available from: http://www.hypertensionaha.org. Accessed February 8, 2019

11. Nair D, Tan S-Y, Gan H-W, et al. The use of ambulatory tonometric radial arterial wave capture to measure ambulatory blood pressure: the validation of a novel wrist-bound device in adults. J Hum Hypertens. 2008;22(3):220-222. doi:10.1038/sj.jhh.1002306

12. Mancia G, Fagard R, Narkiewicz K, et al. 2013 ESH/ESC practice guidelines for the management of arterial hypertension. Blood Press. 2014;23(1):3-16. doi:10.3109/08037051.2014.868629

13. Perinetti G. StaTips Part IV: selection, interpretation and reporting of the intraclass correlation coefficient. South Eur J Orthod Dentofac Res. 2018;5(1). doi:10.5937/sejodr5-17434

14. Giavarina D. Understanding Bland Altman analysis. Biochem Medica. 2015;25(2):141-151. doi:10.11613/BM.2015.015

15. Theilade S, Joergensen C, Persson F, Lajer M, Rossing P. Ambulatory tonometric blood pressure measurements in patients with diabetes. Diabetes Technol Ther. 2012;14(6):453-456. doi:10.1089/dia.2012. 0006

16. Komori T, Eguchi K, Hoshide S, Williams B, Kario K. Comparison of wrist-type and arm-type 24-h blood pressure monitoring devices for ambulatory use. Blood Press Monit. 2013;18(1):57-62. doi:10. 1097/MBP.0b013e32835d124f

17. Agarwal R, Tu W. Minimally sufficient numbers of measurements for validation of 24-hour blood pressure monitoring in chronic kidney disease. Kidney Int. 2018;94:1199-1204. doi:10.1016/j.kint.2018.08. 021
Integrated Blood Pressure Control

\section{Publish your work in this journal}

Integrated Blood Pressure Control is an international, peer-reviewed open-access journal focusing on the integrated approach to managing hypertension and risk reduction. Treating the patient and comorbidities together with diet and lifestyle modification and optimizing healthcare resources through a multidisciplinary team approach constitute key features of the journal. This journal is indexed on

\section{Dovepress}

American Chemical Society's Chemical Abstracts Service (CAS) The manuscript management system is completely online and includes a very quick and fair peer-review system, which is all easy to use. Visit http://www.dovepress.com/testimonials.php to read real quotes from published authors. 